\title{
Variable rate ventilation strategies for the injured lung
}

Gerard L. Gebber PhD, Susan M. Barman PhD

A

LVEOLAR collapse is a negative consequence of monotonous mechanical ventilation (fixed rate and fixed low tidal volume) in patients with acute respiratory distress syndrome (ARDS). By varying the rate at which the mechanical ventilator is driven and the volume delivered, Mutch and colleagues ${ }^{1-3}$ demonstrated that arterial oxygenation in an animal model of ARDS is improved over that attained with monotonous mechanical ventilation. It has been suggested ${ }^{4}$ that the variability of peak airway pressure recruits collapsed alveoli, thereby leading to an increase in $\mathrm{PaO}_{2}$. In this issue of the Journal, Froehlich et al. ${ }^{5}$ have gone one step further by asking whether the temporal order of the fluctuations in breathing rate and volume, as well as the fluctuations themselves, account for the beneficial effects of variable mechanical ventilation. The question arises from the fact that fluctuations in respiratory period and tidal volume, characterizing spontaneous breathing in healthy humans and other species, are time-scale invariant ${ }^{6-8}$ rather than random in occurrence. Time-scale invariant (i.e., fractal) behaviour means that the fluctuations in the value of the measured parameter occurring on one time scale are proportional to some power to those occurring on yet longer time scales. ${ }^{9,10}$ As such, fractal behaviour reflects the linkage via nonlinear interactions of processes occurring on different time scales, thereby leading to long-range correlations among events. As a result, the current value of the measured parameter is influenced not only by recent events, but also by those occurring in the "distant" past. Such longrange correlations are viewed as a type of memory that may be useful in predicting the future behaviour of the respiratory system, and valuable in providing clues to the underlying dynamics governing the respiratory generator and its feedback controllers.
In the current study, Froehlich et al. ${ }^{5}$ used two types of computer-generated files to control the mechanical ventilation of pigs subjected to acute lung injury by the intravenous infusion of oleic acid. One of the files was physiologically-derived from the spontaneous breathing pattern of a healthy human at rest. This file contained long-range fractal correlations of the fluctuations in ventilatory rate. The other file was a white noise file with the same mean value and standard deviation, but with no correlations among events (current value of ventilatory rate is unrelated to past values). Thus, the objective was to determine whether the fractal ordering of events in the physiologically-derived file plays a role in reversing the effects of oleic acid-induced lung injury on $\mathrm{PaO}_{2}$ and other respiratory parameters. For both types of files, the fluctuations in interbreath interval were linked to reciprocal changes in tidal volume, so that minute ventilation was fixed.

The authors used relative dispersion (RD) analysis $^{11}$ and second moment diffusion analysis ${ }^{12}$ to clearly distinguish between the fractal properties of the physiologically-derived file and the lack of correlations in the white noise file used to drive the mechanical ventilator. In the case of $\mathrm{RD}$, a measure of the variance of the breathing rate $(R D=$ mean $/$ standard deviation) is calculated for groups of data points of a specific number $(m)$. Calculation of $\mathrm{RD}$ is repeated each time $m$ is increased. When plotted on a log-log scale, the relationship between $\mathrm{RD}$ and $m$ appears as a straight line (power law) with a negative slope. For a random process (white noise), the power law has a slope of -0.5 . For a fractal process, the power law extends over more than one decade of $m$ values and has a negative slope significantly different from -0.5 .

Following oleic acid-induced lung injury, the ani-

CAN J ANESTH 2008 / 55: 9 / pp 572-576

From the Department of Pharmacology \& Toxicology, Michigan State University, East Lansing, Michigan, USA. Address correspondence to: Dr. Gerard L. Gebber, Department of Pharmacology \& Toxicology, Michigan State University, East Lansing, Michigan 48824, USA. Phone: 517-353-8842; Fax: 517-353-8915; E-mail: gebber@msu.edu Competing interests: None declared. 
mal was mechanically ventilated by using either the physiologically-derived file or the white noise file, and changes in $\mathrm{PaO}_{2}, \mathrm{PaCO}_{2}$, and compliance were followed over a four-hour period. As shown by the plots in Figure 4, the degree and time course of "recovery" of these parameters from the injurious effects of oleic acid infusion did not depend on the file being used to drive the mechanical ventilator. Thus, the authors concluded that the fractal ordering of events provided no advantage over uncorrelated noise. This is a novel field of investigation with potentially important therapeutic implications. At the same time, for reasons given below, the authors' conclusion should be interpreted with some caution.

First, by comparison to the results previously reported by this group, ${ }^{3}$ the degree to which variable mechanical ventilation reversed oleic-induced changes in blood gases and lung compliance in the current study was less impressive. In fact, it is unclear whether either mode (fractal noise or white noise) of variable mechanical ventilation resulted in values of $\mathrm{PaO}_{2}, \mathrm{PaCO}_{2}$, and lung compliance significantly different from those measured immediately after oleic acid-induced lung injury (Figure 4 ). If not, the usefulness of this study would be diminished.

Second, as acknowledged by the authors, the appropriateness of using a physiologically-derived file from a species (human) different from that of the experimental animals can be questioned. Regarding this point, the authors have not tested whether the spontaneous breathing pattern of healthy pigs is characterized by fractal fluctuations in rate and amplitude. Assuming that such is the case, it should be determined whether oleic acid-induced lung injury leads to a breakdown of long-range correlations. If not, the rationale for adding fractal correlated noise to files used to drive the mechanical ventilator would be obscure. Regarding this issue, breakdown of fractal features characterizing normal human heart rate dynamics is known to occur in some cases of heart failure and in atrial fibrillation. ${ }^{13}$

Third, the authors indicate that some of the properties of natural breathing were not captured in the physiologically-derived file used to drive the mechanical ventilator. True physiological variability would include fluctuations in minute volume, I:E ratio, and inspiratory flow rate. These parameters remained fixed for the two modes of variable mechanical ventilation delivered to the animals. Use of a file that more faithfully replicates spontaneous breathing might yield beneficial effects in the management of ARDS.
Future studies are warranted, using surrogates of the physiologically-derived file in addition to, or in place of, the computer-generated white noise file. Surrogate files are created by assigning random numbers to the original data points and then sorting the random numbers by size. ${ }^{14}$ This provides a data set with no correlations whose individual values, as well as mean and standard deviation, are identical to those of the original file. Although the data sets used in the current study were similar, the distributions of individual data points for the two files were not identical (Figure 1).

In summary, the benefits of variable mechanical ventilation over monotonous mechanical ventilation in managing oleic acid-induced lung injury were found not to be dependent on the presence of longrange correlations of the fluctuations in ventilatory rate and volume. It remains to be determined whether a beneficial role of fractal dynamics can be demonstrated with computer-generated files that more faithfully replicate the pattern of normal breathing. For the moment, however, efforts to optimize the beneficial effects of variable mechanical ventilation should be focused as much on changing the distribution of data points comprising the computer-generated file used to drive the mechanical ventilator, as on changing the fractal exponents characterizing their temporal order of occurrence.

\section{Stratégies de ventilation à débit variable pour le poumon blessé}

Le collapsus alvéolaire est une conséquence négative de la ventilation mécanique monotone (débit fixe et volume courant bas fixe) chez les patients souffrant du syndrome de détresse respiratoire aiguë (SDRA). En variant le débit du respirateur mécanique et le volume diffusé, Mutch et coll. ${ }^{1-3}$ ont démontré que l'oxygénation artérielle dans un modèle animal de SDRA est améliorée par rapport à celle obtenue par ventilation mécanique monotone. Il a été suggéré ${ }^{4}$ que la variabilité de la pression maximale du conduit aérien régénère les alvéoles écrasées, permettant ainsi 
une augmentation de $\mathrm{PaO}_{2}$. Dans ce numéro du Journal, Froehlich et coll. ${ }^{5}$ vont un peu plus loin en se demandant si l'ordre temporel des fluctuations dans le débit et le volume respiratoires, outre les fluctuations en soi, sont responsables des effets bénéfiques de la ventilation mécanique variable. La question provient du fait que les fluctuations de période respiratoire et de volume courant, qui caractérisent la respiration spontanée chez les humains et les autres espèces en bonne santé, surviennent de façon invariante plutôt qu'aléatoire à l'échelle du temps ${ }^{6-8} \mathrm{Un}$ comportement invariant à l'échelle du temps (c.-à-d. fractal) signifie que les fluctuations de la valeur du paramètre mesuré survenant sur une échelle de temps sont proportionnelles dans une certaine mesure à celle survenant sur des échelles de temps plus longues. ${ }^{9,10}$ En tant que tel, le comportement fractal reflète la liaison via des interactions non linéaires de processus survenant sur différentes échelles de temps, ce qui mène à des corrélations à long terme entre les divers événements. Par conséquent, la valeur actuelle du paramètre mesuré est influencée non seulement par les événements récents, mais aussi par ceux survenus dans un passé « distant». De telles corrélations à long terme sont considérées comme une sorte de mémoire qui peut être utile pour prévoir le comportement futur du système respiratoire, et précieuse pour fournir des pistes éclairant la dynamique sous-jacente qui régit le générateur respiratoire et ses contrôleurs asservis.

Dans l'étude présentée ici, Froehlich et coll. ${ }^{5}$ ont utilisé deux types de fichiers créés par ordinateur pour contrôler la ventilation mécanique chez des cochons soumis à une lésion pulmonaire aiguë par perfusion intraveineuse d'acide oléique. Un des fichiers se voulait physiologique et était dérivé du schéma de respiration spontanée d'un être humain sain au repos. Ce fichier contenait des corrélations fractales à long terme des fluctuations du débit respiratoire. L'autre était un fichier de bruit blanc avec les mêmes valeur moyenne et écart type, mais sans corrélations entre les événements (c.-à-d., la valeur actuelle du débit respiratoire n'était pas associée à des valeurs passées). Ainsi, l'objectif était de déterminer si l'ordre fractal des événements dans le fichier à base physiologique joue un rôle dans l'inversion des effets d'une lésion pulmonaire provoquée par l'acide oléique sur la $\mathrm{PaO}_{2}$ et d'autres paramètres respiratoires. Pour les deux types de fichiers, les fluctuations dans les intervalles entre les respirations étaient associées à des changements réciproques du volume courant, de telle façon à ce que la ventilation minute soit fixe.
Les auteurs se sont servis d'une analyse par dispersion relative $(D R)^{11}$ et d'une analyse de diffusion du moment du second ordre ${ }^{12}$ pour établir une distinction claire entre les propriétés fractales du fichier d'origine physiologique et l'absence de corrélations dans le fichier de bruit blanc utilisé pour entraîner le respirateur mécanique. Dans le cas de la DR, une mesure de la variance du débit respiratoire $(\mathrm{DR}=$ écart type/moyen) est calculée pour des groupes de points de données d'un nombre spécifique $(m)$. Le calcul de la DR est répété à chaque fois que $m$ est augmenté. Lorsqu'elle est représentée graphiquement sur une échelle logarithmique, la relation entre la DR et $m$ prend la forme d'une ligne droite (loi de puissance) avec une pente négative. Dans le cas d'un processus aléatoire (bruit blanc), la loi de puissance a une pente de $-0,5$. Dans le cas d'un processus fractal, la loi de puissance va au-delà de plus d'une décennie de valeurs $m$ et a une pente négative considérablement différente de -0.5 .

Après avoir été soumis à une lésion pulmonaire provoquée par de l'acide oléique, les animaux ont été ventilés mécaniquement soit avec le fichier physiologique, soit avec le fichier de bruit blanc, et les changements dans la $\mathrm{PaO}_{2}$, la $\mathrm{PaCO}_{2}$ et la compliance pulmonaire ont été monitorés sur une période de quatre heures. Comme les parcelles de la Figure 4 le montrent, le degré et décours temporel de la "récupération» de ces paramètres des effets blessants de la perfusion d'acide oléique n'étaient pas dépendants du fichier utilisé pour alimenter le ventilateur mécanique. Par conséquent, les auteurs ont conclu que l'ordre fractal des événements n'a pas procuré d'avantage par rapport au bruit non corrélé. Il s'agit ici d'un nouveau domaine de recherche qui pourrait potentiellement avoir d'importantes répercussions thérapeutiques. Pourtant, pour les raisons énumérées ci-dessous, la conclusion des auteurs devrait être interprétée prudemment.

En premier lieu, si l'on compare ces résultats à ceux obtenus précédemment par ce même groupe ${ }^{3}$ le degré d'inversion des changements provoqués par l'acide oléique au niveau des gaz sanguins et de la compliance pulmonaire obtenu grâce à la ventilation mécanique variable était moins impressionnant. En fait, nous ne sommes pas certains qu'un mode ou l'autre (bruit fractal ou bruit blanc) de ventilation mécanique variable a donné des valeurs de $\mathrm{PaO}_{2}$, de $\mathrm{PaCO}_{2}$ et une compliance pulmonaire significativement différentes des valeurs mesurées immédiatement après la lésion pulmonaire provoquée par acide oléique (Figure 4). Si ce n'est pas le cas, l'utilité de cette étude serait amoindrie. 
Deuxièmement, et les auteurs le reconnaissent, la décision d'utiliser un fichier physiologique tiré d'une espèce (l'être humain) autre que celle des animaux à l'étude peut être remise en question. Les auteurs n'ont pas mené de test pour déterminer si le schéma de respiration spontanée d'un cochon sain est lui aussi caractérisé par des fluctuations fractales au niveau du débit et de l'amplitude. Présumant que tel serait le cas, il faudrait déterminer si les lésions pulmonaires provoquées par acide oléique engendrent un découpage des corrélations à long terme. Si ce n'est pas le cas, la raison justifiant l'ajout de bruit fractal corrélé aux fichiers utilisés pour alimenter le respirateur mécanique serait obscure. À ce sujet, nous savons qu'un découpage des traits fractals caractérisant la dynamique de fréquence cardiaque humaine normale survient dans certains cas d'insuffisance cardiaque et de fibrillation auriculaire. ${ }^{13}$

Troisièmement, les auteurs indiquent que certaines des propriétés de la respiration naturelle n’étaient pas enregistrées dans le fichier physiologique utilisé pour entraîner le respirateur mécanique. Une variabilité physiologique authentique inclurait des fluctuations du volume minute, un taux I : E, et un débit d'air inspiré. Ces paramètres étaient fixes dans les deux modes de ventilation mécanique variable auxquels les animaux étaient soumis. L'utilisation d'un fichier qui reproduirait plus fidèlement la respiration spontanée pourrait avoir des effets bénéfiques pour la prise en charge du SDRA.

Dès lors, d'autres études sont justifiées, qui utiliseraient des substituts du fichier d'origine physiologique en plus de, ou à la place du fichier de bruit blanc créé par ordinateur. Les fichiers de substitution sont créés en attribuant des nombres aléatoires aux points de données originaux et en classant les nombres aléatoires par ordre de grandeur. ${ }^{14}$ De cette manière, on obtient un ensemble de données sans corrélations dont les valeurs individuelles, ainsi que l'écart type et l'écart moyen, sont identiques à celles du fichier original. Bien que les ensembles de données utilisés dans l'étude en question étaient similaires, les distributions des points de donnée individuels n'étaient pas identiques dans les deux fichiers (Figure 1).

En résumé, il a été observé que les avantages de la ventilation mécanique variable par rapport à la ventilation mécanique monotone pour prendre en charge une lésion pulmonaire provoquée par l'acide oléique ne dépendent pas de la présence de corrélations à long terme des fluctuations du débit et du volume respiratoires. Il reste à déterminer si un rôle bénéfique de la dynamique fractale peut être démontré avec des fichiers créés par ordinateur qui reproduisent de façon plus fidèles le schéma de la respiration normale. Pour le moment, toutefois, les efforts visant à optimiser les effets bénéfiques d'une ventilation mécanique variable devraient se concentrer aussi bien sur le changement de distribution des points de données qui constituent le fichier créé par ordinateur utilisé pour entraîner le respirateur mécanique que sur le changements des exposants fractals qui caractérisent leur ordre temporel d'apparition.

\section{References}

1 Mutch WA, Harms S, Graham MR, et al.

Biologically variable or naturally noisy mechanical ventilation recruits atelectatic lungs. Am J Respir Crit Care Med 2000; 162: 319-23.

2 Boker A, Graham MR, Walley KR, et al. Improved arterial oxygenation with biologically variable or fractal ventilation using low tidal volumes in a porcine model of acute respiratory distress syndrome. Am J Respir Crit Care Med 2002; 165: 456-62.

3 Funk DJ, Graham MR, Girling LG, et al. A comparison of biologically variable ventilation to recruitment manoeuvres in a porcine model of acute lung injury. Respir Res 2004; 5: 22.

4 Suki B, Alencor AM, Sujeer $M K$, et al. Life-support system benefits from noise. Nature 1998; 393: 127-8.

5 Froeblich KF, Graham MR, Buchman TG, et al. Physiological noise versus white noise to drive a variable ventilator in a porcine model of lung injury. Can J Anesth 2008, 55: 577-86.

6 Hoop B, Kazemi H, Liebovitch LS. Rescaled range analysis of resting respiration. Chaos 1993; 3: 27-9.

7 Peng CK, Mietus JE, Liu Y, et al. Quantifying fractal dynamics of human respiration: age and gender effects. Ann Biomed Eng 2002; 30: 683-92.

8 Fadel PJ, Barman SM, Phillips SW, Gebber GL. Fractal fluctuations in human respiration. J Appl Physiol 2004; 97: 2056-64.

9 Feder J. Fractals. New York: Plenum; 1988.

10 Bassingthwaighte JB, Liebovitch LS, West BJ. Fractal Physiology. New York: Oxford University Press, 1994.

11 Bassingthwaighte JB, Raymond GM. Evaluation of the dispersional analysis method for fractal time series. Ann Biomed Eng 1995; 23: 491-505.

12 Glenny $R W$, Robertson, HT, Yamashiro S, Bassingthwaighte JB. Applications of fractal analysis to physiology. J Appl Physiol 1991; 70: 2351-67.

13 Goldberger AL, Peng CK, Hausdorff J, et al. Fractals 
and the heart. In: by Iannaconne PM, Khokha M (Eds). Fractal Geometry in Biological Systems: An Analytical Approach. Raton, FL: CRC Press; 1996: 249-66.

14 Gebber GL, Orer HS, Barman SM. Fractal noises and motions in time series of presympathetic and sympathetic neural activities. J Neurophysiol 2006; 95 : 1176-84.

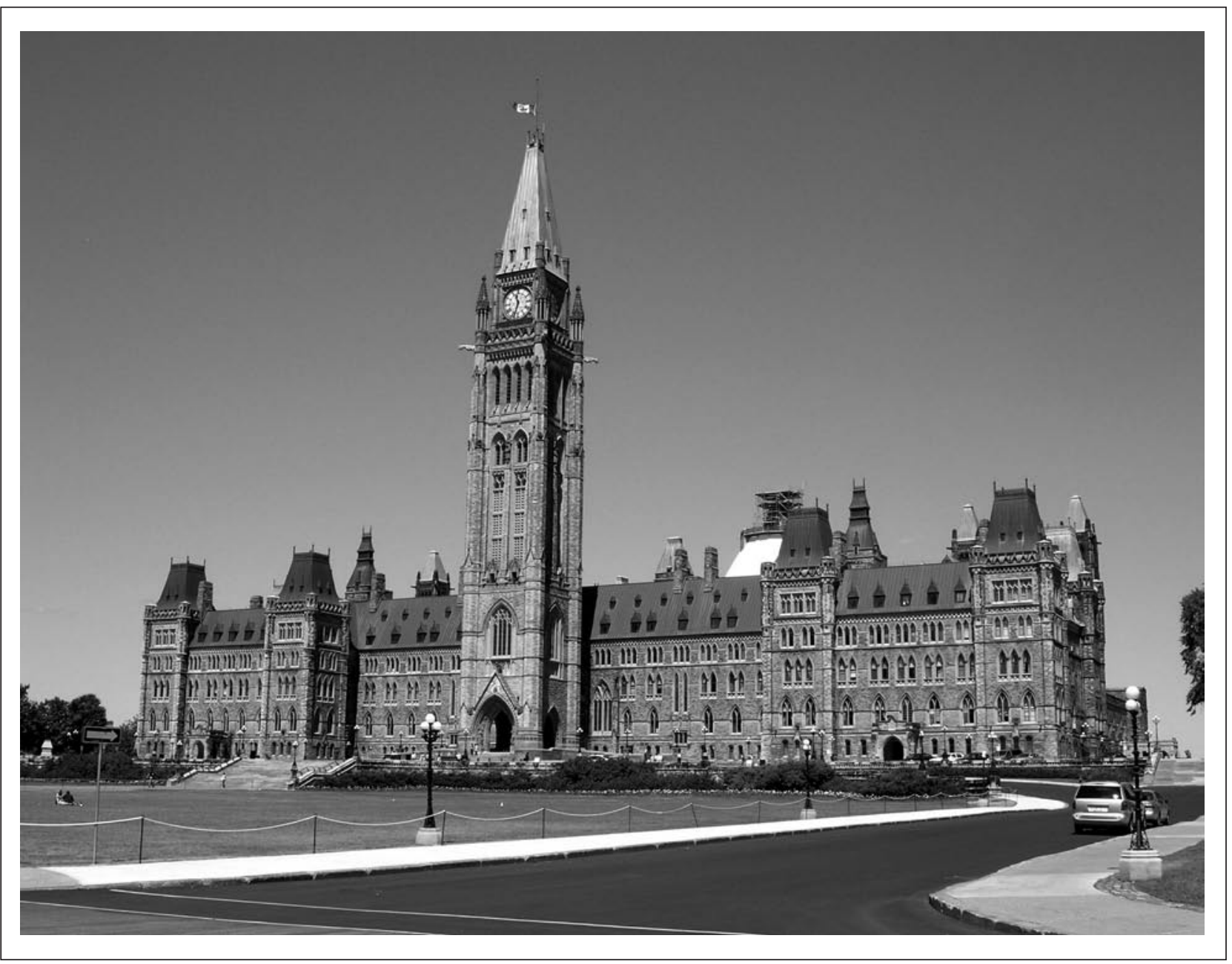

Parliament Buildings - Ottawa 